\title{
A CONTINUED FRACTION OF RAMANUJAN
}

\author{
M. D. HIRSCHHORN
}

(Received 16 March; revised 1 May, 1979)

Communicated by A. J. van der Poorten

\begin{abstract}
In a manuscript discovered in 1976 by George E. Andrews, Ramanujan states a formula for a certain continued fraction, without proof. In this paper we establish formulae for the convergents to the continued fraction, from which Ramanujan's result is easily deduced.
\end{abstract}

1980 Mathematics subject classification (Amer. Math. Soc.): 10 A 32, 10 A 35, 30 B 70, 40 A 15.

In 1976, George E. Andrews discovered a manuscript of Ramanujan (1920?) containing more than six hundred identities. (For the interesting details of this discovery, see Andrews (1979).) One of these identities concerns the curious continued fraction

$$
F(a, b, \lambda, q)=1+\frac{a q+\lambda q}{1+} \frac{b q+\lambda q^{2}}{1+} \frac{a q^{2}+\lambda q^{3}}{1+} \frac{b q^{2}+\lambda q^{4}}{1+} \ldots .
$$

Ramanujan states without proof that

THEOREM 1.

$$
F(a, b, \lambda, q)=\frac{G(a, b, \lambda)}{G(a q, b, \lambda q)}
$$

where

$$
G(a, b, \lambda)=\sum_{n \geqslant 0} \frac{q^{\frac{t\left(n^{2}+n\right)}{}(a+\lambda) \ldots\left(a+\lambda q^{n-1}\right)}}{(1-q) \ldots\left(1-q^{n}\right)(1+b q) \ldots\left(1+b q^{n}\right)}
$$

Andrews (1979) proves this result directly, though with some difficulty. In this 
note we establish formulae for the convergents to $F(a, b, \lambda, q)$, from which Theorem 1 follows easily.

Before proving Theorem 1, we note that applying Watson's theorem ('Watson's $q$-analogue of Whipple's theorem') Watson (1929) to the numerator and denominator of (1.2) yields

\section{THEOREM 2.}

$$
F(a, b, \lambda, q)=\frac{1+\sum_{r \geqslant 1} \frac{\left(1-\lambda q^{2 r}\right)}{\left(1-\lambda q^{r}\right)} \frac{(-\lambda / b)_{r}}{(-b q)_{r}} \frac{(-\lambda / a)_{r}}{(-a q)_{r}} \frac{(\lambda q)_{r}}{(q)_{r}} q^{\frac{1}{2}\left(3 r^{2}+r\right)}(-a b)^{r}}{\sum_{r \geqslant 0}\left(1-\lambda q^{2 r+1}\right) \frac{(-\lambda q / b)_{r}}{(-b q)_{r}} \frac{(-\lambda / a)_{r}}{(-a q)_{r+1}} \frac{(\lambda q)_{r}}{(q)_{r}} q^{\frac{1}{2}\left(3 r^{2}+3 r\right)}(-a b)^{r}}
$$

Theorem 2 contains as corollaries several elegant continued fractions, all given by Ramanujan in Ramanujan (1920?), some of which have appeared previously in the literature. Thus,

$$
\begin{aligned}
& 1+\frac{q}{1+} \frac{q^{2}}{1+} \ldots=\prod_{n \geqslant 0} \frac{\left(1-q^{5 n+2}\right)\left(1-q^{5 n+3}\right)}{\left(1-q^{5 n+1}\right)\left(1-q^{5 n+4}\right)} \text { Rogers (1894), p. 328, Ramanujan (1919), } \\
& 1+\frac{q}{1-} \frac{q-q^{2}}{1+} \frac{q^{3}}{1-} \frac{q^{2}-q^{4}}{1+} \frac{q^{5}}{1-} \ldots=1 / \sum_{n \geqslant 0}(-1)^{n} q^{\frac{i}{2}\left(n^{2}+n\right)}, \quad \text { Eisenstein (1844), } \\
& 1+\frac{q+q^{2}}{1+} \frac{q^{2}+q^{4}}{1+} \frac{q^{3}+q^{6}}{1+} \ldots=\prod_{n \geqslant 0} \frac{\left(1-q^{6 n+3}\right)^{2}}{\left(1-q^{6 n+1}\right)\left(1-q^{6 n+5}\right)}, \\
& 1+\frac{q}{1+} \frac{q+q^{2}}{1+} \frac{q^{3}}{1+} \frac{q^{2}+q^{4}}{1+} \frac{q^{5}}{1+} \ldots=\prod_{n \geqslant 0} \frac{\left(1-q^{4 n+2}\right)^{2}}{\left(1-q^{4 n+1}\right)\left(1-q^{4 n+3}\right)}, \\
& 1+\frac{q+q^{2}}{1+} \frac{q^{4}}{1+} \frac{q^{3}+q^{6}}{1+} \frac{q^{8}}{1+} \ldots=\prod_{n \geqslant 0} \frac{\left(1-q^{8 n+3}\right)\left(1-q^{8 n+5}\right)}{\left(1-q^{8 n+1}\right)\left(1-q^{8 n+7}\right)},
\end{aligned}
$$

and

Ramanujan (1920 ?),

$$
1-\frac{q-q^{2}}{1-} \frac{q^{2}-q^{4}}{1-} \frac{q^{3}-q^{6}}{1-} \ldots=1 / \sum_{n \geqslant 0}(-1)^{n} q^{3 n^{2}+2 n}\left(1+q^{2 n+1}\right),
$$

Ramanujan (1920 ?). 
Our main result, proved in Section 3, is

THEOREM 3.

$$
\begin{aligned}
& 1+\frac{a q+\lambda q}{1+\underline{b q+\lambda q^{2}}} \quad=\frac{P_{2 N-1}(a, b, \lambda)}{P_{2 N-2}(b, a q, \lambda q)}, \\
& \overline{\frac{1+a q^{N}+\lambda q^{2 N-1}}{1}}
\end{aligned}
$$

and

$$
\begin{aligned}
& 1+\frac{a q+\lambda q}{1+\frac{b q+\lambda q^{2}}{1+}} \\
& =\frac{P_{2 N}(a, b, \lambda)}{P_{2 N-1}(b, a q, \lambda q)}, \\
& \overline{1+\frac{b q^{N}+\lambda q^{2 N}}{1}}
\end{aligned}
$$

where

$$
\begin{aligned}
P_{N}(a, b, \lambda)=\sum a^{s} b^{t} \lambda^{u} q^{\Delta(s+t)+s u+t u+u^{2}} & \\
\times & {\left[\begin{array}{c}
N+1-s-t-u \\
u
\end{array}\right]\left[\begin{array}{c}
{[(N+1) / 2]-t-u} \\
s
\end{array}\right]\left[\begin{array}{c}
{[N / 2]-s-u} \\
t
\end{array}\right], }
\end{aligned}
$$

the sum being taken over all $s, t, u \geqslant 0$ such that $s+t+u \leqslant[(N+1) / 2]$, for our present purposes $\left[\begin{array}{c}-1 \\ 0\end{array}\right]=1$, and $\Delta(n)=\frac{1}{2}\left(n^{2}+n\right)$.

Letting $N \rightarrow \infty$ in (2.1) and (2.2), we obtain

$$
F(a, b, \lambda, q)=\frac{P(a, b, \lambda)}{P(b, a q, \lambda q)},
$$

where

$$
P(a, b, \lambda)=\sum_{s, t, u \geqslant 0} a^{s} b^{t} \lambda^{u} \frac{q^{\Delta(s+t)+s u+t u+u^{2}}}{(q)_{s}(q)_{t}(q)_{u}} .
$$

It is obvious from (2.4) that

$$
P(a, b, \lambda)=P(b, a, \lambda) .
$$

Also

$$
P(a, b, \lambda)=\prod_{n \geqslant 1}\left(1+b q^{n}\right) . G(a, b, \lambda), .
$$


where $G(a, b, \lambda)$ is given by (1.3). For,

$$
\begin{aligned}
& P(a, b, \lambda)=\sum_{s, t, u \geqslant 0} a^{s} b^{t} \lambda^{u} \frac{q^{\Delta(s)+\Delta(t)+s t+s u+t u+u^{2}}}{(q)_{s}(q)_{t}(q)_{u}} \\
& =\sum_{s, u \geqslant 0} a^{s} \lambda^{u} \frac{q^{\Delta(s)+s u+u^{2}}}{(q)_{s}(q)_{u}} \sum_{t \geqslant 0} \frac{q^{\Delta(t)}\left(b q^{s+u}\right)^{t}}{(q)_{t}} \\
& =\sum_{s, u \geqslant 0} a^{s} \lambda^{u} \frac{q^{\Delta(s)+s u+u^{2}}}{(q)_{s}(q)_{u}}\left(1+b q^{s+u+1}\right)\left(1+b q^{s+u+2}\right) \ldots \\
& =\prod_{n \geqslant 1}\left(1+b q^{n}\right) \sum_{s, u \geqslant 0} a^{s} \lambda^{u} \frac{q^{\Delta(s)+s u+u^{2}}}{(q)_{s}(q)_{u}(1+b q) \ldots\left(1+b q^{s+u}\right)} \\
& =\prod_{n \geqslant 1}\left(1+b q^{n}\right) \sum_{n \geqslant 0} \frac{q^{\Delta(n)}}{(q)_{n}(1+b q) \ldots\left(1+b q^{n}\right)} \times \sum_{s+u=n} a^{s} \lambda^{u} q^{\Delta(u-1)}\left[\begin{array}{l}
n \\
u
\end{array}\right] \\
& =\prod_{n \geqslant 1}\left(1+b q^{n}\right) \sum_{n \geqslant 0} \frac{q^{\Delta(n)}(a+\lambda) \ldots\left(a+\lambda q^{n-1}\right)}{(q)_{n}(1+b q) \ldots\left(1+b q^{n}\right)} \\
& =\prod_{n \geqslant 1}\left(1+b q^{n}\right) G(a, b, \lambda) .
\end{aligned}
$$

From (2.3), (2.5) and (2.6) it follows that

which is (1.2).

$$
F(a, b, \lambda, q)=\frac{P(a, b, \lambda)}{P(a q, b, \lambda q)}=\frac{G(a, b, \lambda)}{G(a q, b, \lambda q)},
$$

We establish Theorem 3 by showing that if $P_{N}(a, b, \lambda)$ is defined by (2.2), then

$$
P_{0}=1, \quad P_{1}=1+a q+\lambda q
$$

and

$$
P_{N}(a, b, \lambda)=P_{N-1}(b, a q, \lambda q)+(a q+\lambda q) P_{N-2}\left(a q, b q, \lambda q^{2}\right) .
$$

We can write (3.2)

$$
\frac{P_{N}(a, b, \lambda)}{P_{N-1}(b, a q, \lambda q)}=1+\frac{(a q+\lambda q)}{\left(\frac{P_{N-1}(b, a q, \lambda q)}{P_{N-2}\left(a q, b q, \lambda q^{2}\right)}\right)} .
$$

Theorem 3 follows by iteration of (3.3), together with (3.1). 
ProOF OF (3.2). Write

$$
P_{N}(a, b, \lambda)=\sum a^{s} b^{t} \lambda^{u} q^{f(s, t, u)} c_{N}(s, t, u),
$$

where

$$
f(s, t, u)=\Delta(s+t)+s u+t u+u^{2}
$$

and

$$
c_{N}(s, t, u)=\left[\begin{array}{c}
N+1-s-t-u \\
u
\end{array}\right]\left[\begin{array}{c}
{[(N+1) / 2]-t-u} \\
s
\end{array}\right]\left[\begin{array}{c}
{[N / 2]-s-u} \\
t
\end{array}\right]
$$

Then

$$
\begin{gathered}
f(t, s, u)=f(s, t, u), \\
s+t+u+f(s-1, t, u)=f(s, t, u), \\
s+t+2 u-1+f(s, t, u-1)=f(s, \dot{t}, u)
\end{gathered}
$$

and

(3.8) $c_{N-2}(s, t, u-1)+q^{u}\left(c_{N-2}(s-1, t, u)+q^{s} c_{N-1}(t, s, u)\right)=c_{N}(s, t, u)$.

For,

$$
\begin{aligned}
c_{N-2}(s-1, t, u)+q^{s} c_{N-1}(t, s, u) & =\left[\begin{array}{c}
N-s-t-u \\
u
\end{array}\right]\left[\begin{array}{c}
{[(N-1) / 2]-t-u} \\
s-1
\end{array}\right]\left[\begin{array}{c}
{[N / 2]-s-u} \\
t
\end{array}\right] \\
+ & q^{s}\left[\begin{array}{c}
N-t-s-u \\
u
\end{array}\right]\left[\begin{array}{c}
{[N / 2]-s-u} \\
t
\end{array}\right]\left[\begin{array}{c}
{[(N-1) / 2]-t-u} \\
s
\end{array}\right] \\
= & {\left[\begin{array}{c}
N-s-t-u \\
u
\end{array}\right]\left[\begin{array}{c}
{[N / 2]-s-u} \\
t
\end{array}\right]\left\{\left[\begin{array}{c}
{[(N-1) / 2]-t-u} \\
s-1
\end{array}\right]\right.} \\
& \left.+q^{s}\left[\begin{array}{c}
{[(N-1) / 2]-t-u} \\
s
\end{array}\right]\right\} \\
= & {\left[\begin{array}{c}
N-s-t-u \\
u
\end{array}\right]\left[\begin{array}{c}
{[N / 2]-s-u} \\
t
\end{array}\right]\left[\begin{array}{c}
{[(N+1) / 2]-t-u} \\
s
\end{array}\right] }
\end{aligned}
$$

and so

$$
\begin{aligned}
& c_{N-2}(s, t, u-1)+q^{u}\left(c_{N-2}(s-1, t, u)+q^{s} c_{N-1}(t, s, u)\right) \\
&=\left[\begin{array}{c}
N-s-t-u \\
u-1
\end{array}\right] {\left[\begin{array}{c}
{[(N+1) / 2]-t-u} \\
s
\end{array}\right]\left[\begin{array}{c}
{[N / 2]-s-u} \\
t
\end{array}\right] } \\
&+q^{u}\left[\begin{array}{c}
N-s-t-u \\
u
\end{array}\right]\left[\begin{array}{c}
{[N / 2]-s-u} \\
t
\end{array}\right]\left[\begin{array}{c}
{[(N+1) / 2]-t-u} \\
s
\end{array}\right]
\end{aligned}
$$




$$
\begin{aligned}
& =\left[\begin{array}{c}
{[(N+1) / 2]-t-u} \\
s
\end{array}\right]\left[\begin{array}{c}
{[N / 2]-s-u} \\
t
\end{array}\right]\left\{\left[\begin{array}{c}
N-s-t-u \\
u-1
\end{array}\right]\right. \\
& \left.+q^{u}\left[\begin{array}{c}
N-s-t-u \\
u
\end{array}\right]\right\} \\
& =\left[\begin{array}{c}
N+1-s-t-u \\
u
\end{array}\right]\left[\begin{array}{c}
{[(N+1) / 2]-t-u} \\
s
\end{array}\right]\left[\begin{array}{c}
{[N / 2]-s-u} \\
t
\end{array}\right] \\
& =c_{N}(s, t, u) .
\end{aligned}
$$

It follows from (3.4), (3.7) and (3.8) that

$$
\begin{aligned}
P_{N-1} & (b, a q, \lambda q)+(a q+\lambda q) P_{N-2}\left(a q, b q, \lambda q^{2}\right) \\
= & \sum b^{s} a^{t} \lambda^{u} q^{t+u+f(s, t, u)} c_{N-1}(s, t, u) \\
& +a \sum a^{s} b^{t} \lambda^{u} q^{s+t+2 u+1+f(s, t, u)} c_{N-2}(s, t, u) \\
& +\lambda \sum a^{s} b^{t} \lambda^{u} q^{s+t+2 u+1+f(s, t, u)} c_{N-2}(s, t, u) \\
= & \sum a^{s} b^{t} \lambda^{u} q^{s+u+f(t, s, u)} c_{N-1}(t, s, u) \\
& +\sum a^{s} b^{t} \lambda^{u} q^{s+t+2 u+f(s-1, t, u)} c_{N-2}(s-1, t, u) \\
& +\sum a^{s} b^{t} \lambda^{u} q^{s+t+2 u-1+f(s, t, u-1)} c_{N-2}(s, t, u-1) \\
= & \sum a^{s} b^{t} \lambda^{u} q^{f(s, t, u)} \\
& \times\left\{q^{s+u} c_{N-1}(t, s, u)+q^{u} c_{N-2}(s-1, t, u)+c_{N-2}(s, t, u-1)\right\} \\
= & \sum a^{s} b^{t} \lambda^{u} q^{f(s, t, u)} c_{N}(s, t, u) \\
= & P_{N}(a, b, \lambda),
\end{aligned}
$$

which is (3.2), as required.

\section{Acknowledgement}

I would like to thank $\mathrm{L}$. Yeo for computing $P_{N}$ for $N \leqslant 10$ from (3.2). It was from these that the form (2.2) was constructed.

\section{References}

G. E. Andrews (1979), ‘An introduction to Ramanujan's "lost" notebook', Amer. Math. Monthly 86, 89-108.

G. Eisenstein (1844), 'Transformations remarquables de quelques séries', Crelle's Journal 27, 193-197. 
B. Gordon (1965), 'Some continued fractions of the Rogers-Ramanujan type', Duke Math. J. 32, 741-748.

S. Ramanujan (1919), 'Proof of certain identities in combinatory analysis', Proc. Camb. Phil. Soc. 19, 214-216.

S. Ramanujan (1920?), unpublished manuscript.

L. J. Rogers (1894), 'Second memoir on the expansion of certain infinite products', Proc. London Math. Soc. 25, 318-343.

G. N. Watson, 'A new proof of the Rogers-Ramanujan identities', J. London Math. Soc. 4, 4-9.

School of Mathematics

University of New South Wales

Kensington, NSW 2033

Australia 